\title{
NOD2-associated granulomatous autoinflammatory syndromes - a short update for clinicians
}

\author{
Laura Damian', Mihaela Sparchez ${ }^{2,3}$, Mihaela Lupse ${ }^{4,5}$, Ioana Felea', Simona Rednic ${ }^{1,6}$, \\ Cristina Pamfil ${ }^{1,6}$, Camelia Bucsa ${ }^{7}$, Romana Vulturar ${ }^{8,9}$ \\ ${ }^{1}$ Rheumatology Department, Emergency Clinical County Hospital Cluj, \\ Centre for Rare Autoimmune and Autoinflammatory Diseases (ERN-ReCONNET) \\ ${ }^{2} 2^{\text {nd }}$ Pediatric Department, "Iuliu Hatieganu" University of Medicine and Pharmacy, Cluj-Napoca, Romania \\ ${ }^{3} 2^{\text {nd }}$ Pediatric Department, Emergency Clinical Pediatric Hospital Cluj, Cluj-Napoca, Romania \\ ${ }^{4}$ Department of Infectious Diseases, "Iuliu Hatieganu" University of Medicine and Pharmacy, \\ Cluj-Napoca, Romania \\ ${ }^{5}$ Infectious Diseases Clinical Hospital, Cluj, Cluj-Napoca, Romania \\ ${ }^{6}$ Rheumatology Department, "Iuliu Hatieganu" University of Medicine and Pharmacy, \\ Cluj-Napoca, Romania \\ ${ }^{7}$ Drug Information Research Center, "Iuliu Hatieganu" University of Medicine and Pharmacy, \\ Cluj-Napoca, Romania \\ ${ }^{8}$ Department of Molecular Sciences, "Iuliu Hatieganu" University of Medicine and Pharmacy, \\ Cluj-Napoca, Romania \\ ${ }^{9}$ Cognitive Neuroscience Laboratory, "Babes-Bolyai" University, Cluj Napoca, Romania
}

NOD2 (nucleotide-binding oligomerization domain-2), a pattern recognition receptor, is involved in innate immune defense against pathogens, intestinal mucosal barrier integrity, gut microbiota composition, autophagy, immune homeostasis and inflammation. NOD2 mutations have been associated primarily with childhood diseases (Blau syndrome and sarcoidosis with early-onset, monogenic Crohn's disease), but also with adult diseases (NOD2-associated autoinflammatory syndrome - NAID, also called Yao syndrome etc.). Intermediate forms between Blau syndrome and NAID have also been described. Blau's disease and early-onset sarcoidosis are the familial and the sporadic forms respectively of a dominantly inherited rare monogenic autoinflammatory disease. Blau's syndrome starts in early childhood, evolving with non-caseating granulomatous arthritis with prominent tenosynovitis, dermatitis with a "bronzed", maculo-papular or scaly skin rash, and intermittent fever. Periodic fever, generalized lymphadenopathy, and granulomatous visceral involvement may be present. Therapy consists in systemic steroids, immunosuppressants and biologic drugs. In adults the NAID or Yao's syndrome evolves with bouts of systemic inflammation with lower limbs swelling, tenosynovitis and non-erosive arthritis, fever and dermatitis. We discuss the differential diagnosis with other granulomatous diseases. Increased awareness is necessary regarding these rare diseases which may alter the quality of life or lead to disability, in order to improve their prognosis.

Keywords: Blau syndrome, NAID, NOD2-associated inflammation, granulomatous autoinflammation, periodic fever

\section{INTRODUCTION}

Systemic autoinflammatory diseases (SAID) are a spectrum of clinically and genetically heterogene- ous disorders, evolving with bouts of apparently unprovoked inflammation (1). The last years have brought about a series of new pathogenic entities 
related to granulomatous autoinflammation, in which NOD2 mutations have been found. The clinical features, although orientating, are currently not enough in order to diagnose a certain SAID, as different diseases may have similar presentations. In some cases, the right diagnosis may translate into a better disease management. For instance, Crohn's disease, involving the digestive tract, has an incompletely understood pathogenesis, likely related to an exaggerated inflammatory response directed to the luminal microbiota (2). In the last years, a Crohn's disease clinical picture has been shown to be related from mevalonate kinase deficiency to NOD2-associated diseases and others. Some SAIDassociated genes are located in chromosome 16, such as the familial Mediterranean fever gene MEFV and the NOD2 gene. The use of next generation sequencing (NGS) may uncover several genes involved in inflammation, contributing to the heterogeneity of the clinical picture (1). The new drug development based upon the signaling pathways could advance the personalized therapy (3).

\section{NOD2}

NOD2 (nucleotide-binding oligomerization domain-containing protein 2) is a molecule involved in preservation of the intestinal mucosal barrier integrity and immune homeostasis, proper autophagy and balancing the gut microbiota composition (2). NOD2 is a member of the NOD-like (NLR) family of pattern recognition receptors involved in innate immune defense against pathogens and in inflammation (4). NOD2 shares molecular structure with NOD1, pyrin and cryopirin (3). This protein is expressed mainly in peripheral blood lymphocytes and is involved in immune responses (5). NOD2 functions mainly through RICK or RIP2 to activate p38 mitogen- activated protein kinases and NFkB, resulting in enhanced autophagy and inflammation (3). The NOD2 variants located in the leucine-rich repeat (LRR) region are associated with Crohn's disease, while the variants in the nucleotide-binding domain (NBD) and in between the NBD and LRR are associated with Blau syndrome and the NOD2-associated autoinflammatory disease (NAID), respectively (3).

NOD2 variants have not been described systemic lupus erythematosus, psoriasis or psoriatic arthritis, ankylosing spondylitis, granulomatosis with polyangiitis or adult sarcoidosis (3). Certain gain-offunction NOD2 mutations have been associated with Crohn's disease, including its early-onset variant. Others have been described in Blau's disease and in early-onset sarcoidosis, evolving with granulomas in different affected tissues. The most recent entity, called NOD2-associated autoinflammatory disease (NAID) or Yao's syndrome, described by Yao et al in 2011, affects the adults, mostly females; intermediate forms between Blau's syndrome and NAID have been described as well (6).

Protein NOD2 (figure 1) is also known as caspase recruitment domain-containing protein 15 (CARD15) or inflammatory bowel disease protein 1 (IBD1), is a protein that in humans is encoded by the NOD2 gene located on chromosome 16 . The gene is constituted by multiple exons and introns distributed along $39.5 \mathrm{~Kb}$. The protein contains $1040 \mathrm{ami}-$ no acids. The NOD2 protein is a very dynamic cytoplasmic receptor; once it is activated in the cells, numerous interactions and responses are mediated, and these include inflammasome regulation, production of proinflammatory cytokines, triggering autophagy, production of type I interferons (IFNs), and other antiviral activities (as is activation of RNAse L with viral RNAs degradation) (7).

\section{BLAU'S SYNDROME AND EARLY-ONSET SARCOIDOSIS}

Blau's syndrome (BS) and early-onset sarcoidosis (EOS) are the familial and the sporadic forms respectively of a dominantly inherited rare monogenic autoinflammatory disease (8) (for this reason their description will be common in the following). The pathogenesis of the disease is not well understood. Blau syndrome (and early-onset sarcoidosis) are classically described as being caused by hyperfunctional mutations in the NOD2 gene, but may in fact also be caused by hypofunctional mutations, as in Crohn's disease (9). In a prospective international cohort of BS having recruited 31 patients, the NOD2 mutations observed were the p.R334W, p.R334Q and others (8). Most of the NOD2 mutations described to date are located in exon 4 of the gene (10, 11).

Blau's syndrome (OMIM \#186580) is classically characterized by non-caseating granulomatous arthritis, dermatitis and uveitis (10). BS usually starts in early childhood and its suggestive clinical sign is the presence of a "bronzed", reddish-brown maculo-papular or scaly ichthyosis-like skin rash. Fever attacks may add to the clinical picture.

Classically described as gain-of-function NOD2 mutations, BS/EOS may actually be caused by lossof-function mutations, similar to Crohn's disease (9).

The arthritis in BS/EOS is symmetric and most often polyarticular, but oligoarticular involvement was also possible. The arthritis involved mostly the wrists, ankles, knees and proximal interphalangeal joints, and BS patients may still present active arthritis beyond 10 years (8). Tenosynovitis is prominent, "boggy". Camptodactylia and proximal interphalangeal contracture may also appear. The axial 


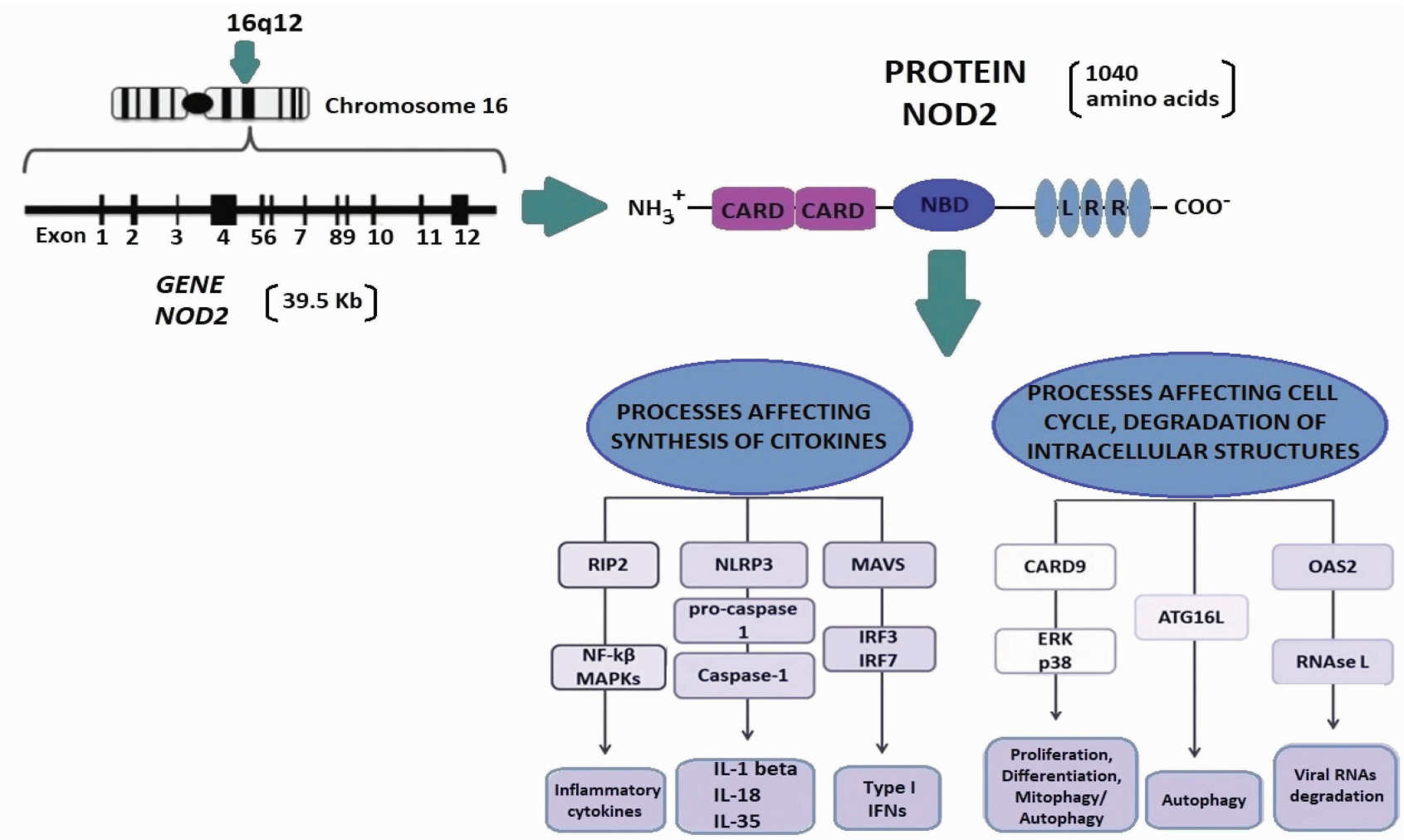

FIGURE 1. Representation of the NOD2 gene and protein with the types of cellular processes triggered by its activation, relevant for immune responses (1,7). LRR - leucine-rich repeat; RIP2 - receptor-interacting protein 2; ERK - extracellular signal-regulated kinase; ATG16L1 - autophagy-related 16-like 1 protein complex; IRF3, IRF7 - interferon regulatory factors 3 and 7; MAPK - mitogen-activated protein kinase; MAVS - mitochondrial antiviral-signaling protein, NF-KB - nuclear factor $\mathrm{KB}$, OAS2 - 2'-5'-oligoadenylate synthetase type 2

skeleton and the temporo-mandibular joints are apparently not involved (4).

Radiologically the arthritis is generally non-erosive, with diffuse periarticular osteopenia. Joint space narrowing, dysplasia of the scaphoid and lunate, with rotation and pseudo-collapse of the lunate and scaphoid bone, and a long thin diaphysis of the second metacarpal bone may be seen as well (8). Of interest, associated biconcave radial or ulnar epiphysis, ulnar shortening and abnormal plump distal ulna are typical of the BS hand in adults (8). The virtual absence of epiphyseal overgrowth may help differentiate the hand in BS from a hand in polyarticular juvenile arthritis (8). The dysplastic bony changes noted in $2 / 3$ of BS patients suggest a role of NOD2 in bone morphogenesis (8).

The ocular disease is frequent, with granulomatous uveitis and vitreal inflammation in $2 / 3$ of cases, leading to visual loss in $1 / 3$ cases (8). The BS uveitis is a progressive panuveitis with multifocal choroiditis (12). Ophtalmic periodic assessments should be performed. The skin disease, apart from the typical "bronzed" or reddish-brown, mildly scaly rash on the trunk and extremities described above, may be very discrete, imitating atopic dermatitis (4). Periodic fever, generalized lymphadenopathy, hepatosplenomegaly and granulomatous hepatitis are quite common. Also, other types of involvement - renal (interstitial nephritis, nephrocalcinosis or hypertension), cardiac, vascular (large-vessel Takayasulike arteritis, leucocytoclastic vasculitis) or neurological (cranial or peripheral neuropathies), have been described $(8,13)$. Other features may include, like in adult's sarcoidosis, erythema nodosum and sialadenitis (8). Unlike the adult-onset sarcoidosis, the lung involvement is not usual (8). Nevertheless, interstitial lung disease was seen in a minority of prospectively followed-up patients, and pulmonary embolism was noted in a case (8).

There is no gold standard therapy for BS/EOS (9). Therapy consists in systemic steroids, immunosuppressive disease-modifying and biologic drugs (anti-TNF, anti-IL-1 etc.). $(8,14)$. The anti-TNF therapy (infliximab, adalimumab and etanercept) was the most commonly used therapy, with only partial control of the articular and ocular disease (8). Canakinumab was also used successfully in a case of BS syndrome with refractory uveitis (15). 


\section{THE NOD2-ASSOCIATED AUTOINFLAMMATORY DISEASE (YAO SYNDROME)}

The NOD2- associated autoinflammatory disease (Yao syndrome or NAID, OMIM \#617321) is a genetically complex multisystem disorder evolving with periodic fever, inflammatory arthritis with "boggy" tenosynovitis affecting mostly the lower legs and dermatitis (1). The estimated incidence is 1-10 cases/100,000 cases (16). The disease affects white adults with a 2:1 female to male ratio (17). Nevertheless, there is also a report of possible co-existance of Blau syndrome and NAID in a child (18).

A flare may last days to weeks, with an asymptomatic interval of several weeks or months. The arthralgia, oligo-or polyarthritis are common, nonerosive, characteristically associated with swelling of the lower extremities and occur periodically; between flares the joints may appear normal. However, $1 / 4$ of patients may develop intermittent unilateral or bilateral lower limbs swelling involving the ankles and feet. Other clinical features are a siccalike syndrome and gastrointestinal involvement (3). Some cases evolve with unexplained recurrent bouts of high fever, abdominal pain, diarrhea, vomiting and non-pruritic erythematous patches on the face and trunk (19). Granulomatous pneumonitis with non-necrotizing granulomas, pleuritis, pericarditis or ocular myositis have been rarely reported $(20,21)$.

The differential diagnosis of NAID is broad, mostly with Blau's syndrome, Crohn's disease or other SAID. Of interest, the paroxysmal episodes of inflammation, with fever and diarrhea, in NAID may mimic a mast cell disorder or a neuroendocrine tumor, but the vasoactive intestinal peptide VIP was increased in a NAID case, suggesting common mechanisms (22).

During an inflammatory episode, the laboratory may show non-specific inflammation (leukocytosis with neutrophilia, anemia). Small titers of antinuclear antibodies may transiently appear (19). The skin biopsy shows a granulomatous dermatitis with many histiocytes or a spongiotic dermatitis (23).

In a large cohort of 143 adult patients, most of them female and Caucasian, with a clinical phenotype suggestive of NAID, almost half of them (67) carried NOD2 variants, and 54 of the 67 carriers (81\%) had NAID, which was sporadic in almost all cases. The genetic profile, distinct from Crohn's disease, NOD2 variants associated were IVS9 $(+158)$ or compound heterozygote IVS8(+158) and R702W.

Of interest, NOD2 expression and pathway activation are aberrant in NAID, and specific NOD2 genotypes result in distinct NOD2 expression and cytokine profiles (17). The dysfunctional NOD2 in NAID was hypothesized to be an amplifier or modi- fier of inflammation, and not necessarily an initiator of inflammation (17).

The therapy in NAID consists in glucocorticoids and sulfasalazine, the mainstay of the therapy during flares. However about $1 / 2$ of patients have frequent flares. Biologic drugs, such as anti-TNFs, IL-6 inhibitors or canakinumab, may be beneficial in refractory cases $(16,17,24)$.

\section{NOD2-ASSOCIATED CROHN'S DISEASE}

NOD2 mutations have been associated to Crohn's disease, and recessive inheritance of NOD2 alleles explain $10 \%$ of the adult CD cases, most of them with early onset (25). The three main Crohn's disease-associated NOD2/CARD15 polymorphisms are p.R702W, p.G908R and p.L1007fs (26). Carriers of more than one allele of NOD2 risk alleles have increased susceptibility to Crohn's disease, while recessive inheritance of biallelic rare and low-frequency NOD2 variants were found in about $8 \%$ in a pediatric Crohn's disease cohort (25). Patients with early-onset Crohn's disease generally have a more severe disease than in older age, with upper gastrointestinal tract involvement, small bowel and perianal disease (25). Moreover, the prevalence of complications such as low bone mineral density is increased in early-onset Crohn's disease (26).

\section{OTHER DISEASES ASSOCIATED WITH NOD2 OR THE NOD2 PATHWAY}

Familial camptodactily associated with granulomatous disease was proposed as an intermediate form of NOD2-assocciated diseases (6). Of note, the patients, two Caucasian women in their 50s, with inflammatory arthritis and a familial history of camptodactily, had NOD2 sequence variants IVS8(+158) and R703C respectively, and non-caseating granuloma either mediastinal or subcutaneous periarticular, and one had parotid enlargement and fever (6). These clinical features suggested that granulomatous disease associated with NOD2 variants may be an intermediate form between Blau and NAID (6).

Interestingly, although NOD2 is considered as not being involved in adult sarcoidosis (3) other NOD2 pathway genes (TAB1, TAB2, MAPK-13) were found in adult sarcoidosis cases with clinical characteristics of BS (26). Therefore, alteration of the NOD2 pathway downstream genes might play a role in the granulomatous inflammation common to BS and sarcoidosis (27).

NOD2 increased mRNA has been found in Behçet's disease (28) and in Vogt-Koyanagi-Harada syndrome, a rare granulomatous inflammatory ocular and central nervous system disease disease 
(29). NOD2 expression was differentially increased in rheumatoid arthritis, in cardiovascular disease, in obesity and metabolic syndrome $(30,31)$. NOD2 polymorphisms have been related to atopic dermatitis, as well (32).

Also, in dental caries and aggressive periodontitis occurs at an early age causing rapid periodontal tissues destruction, and NOD2 variants, among other genes, have been associated with this clinical entity $(5,31)$.

NOD2 polymorphisms (particulary the most common ones R702W, G908R and 3020insC) have been associated with different cancers (gastric, colorectal, ovarian, breast, prostate, testicular, pulmonary, renal, urinary bladder, skin, non-thyroid endocrine tumors, lymphoma and leukemia) $(31,33)$.

\section{MONOGENIC DISEASES MIMICKING NOD2-ASSOCIATED GRANULOMATOUS DISEASE}

Other monogenic diseases with overlapping autoinflammatory, autoimmune and immunodeficiency features, may have similar presentations with those of NOD2-associated autoinflammatory diseases (34). For instance, pathogenic mutations of the PLCG2 gene encoding the phospholipase C (PLCy2), enzyme expressed in hematopoietic cells lead to two diseases, both dominantly inherited: PLAID and APLAID. PLAID (PLCG2-associated antibody deficiency and immune dysregulation) is characterized by skin granuloma formation (on the face, nose, fingers etc.), cold-induced urticaria, sometimes with blisters and various autoimmune and immunodeficiency features (34). APLAID (autoinflamation-PLCy2-associated antibody deficiency- immune deficiency) occurs early in life, evolving with fever, heat- or sweating-induced blisters or rashes, ocular inflammation, enterocolitis, arthralgia and progressive interstitial lung disease $(35,36)$. In PLAID and APLAID, immunoglobulins may be necessary for the immunodeficiency, while the inflammation is currently difficult to treat, as it does not respond to anti-IL-1 or other cytokine inhibitors, suggesting yet undescribed pathogenic pathways involved (34).

The LACC1 gene encodes for the protein FAMIN (fatty acid metabolism and immunity nexus), with homologies to bacterial multicopper oxidoreductases (34). FAMIN, expressed in macrophages, controls the lipid oxidation and lipogenesis. LACC1 is involved in pathogen clearance and in granulomatous disease, similar to NOD2 (34). LACC1 mutations were associated with early-onset Crohn's disease, systemic juvenile idiopathic arthritis and dissemi- nated leprosy, implying a role of the environmental factory, including microbiota, in the disease expression (34). Also, the study of phenotypes associated with NOD2 and LACC1 mutations underscores the role of environmental factors in modulating the clinical picture in more common diseases, such as sarcoidosis (9).

Also, disseminated granulomas may appear in chronic granulomatous disease, a rare primary immune deficiency due to mutations of the phagocytic NADPH oxidase genes (CYBB, CYBA, NCF1, NCF2 and NCF4). The disease is characterized by recurrent, life-threatening bacterial and fungal infections of the skin, lymph nodes, lungs, liver, kidneys, bones and brain (37). Excessive inflammation, including colitis and perirectal abscesses mimicking Crohn's disease, may parallel this immune deficiency. Arthritis, uveitis, glomerulonephritis or lupus may add to the clinical picture. The diagnosis is made by measuring the NADPH oxidase activity, for instance with the nitroblue tetrazolium (NBT) test. Genetic testing (including for G6PD deficiency and Rac2 genes mutation leading to similar clinical pictures) should be performed whenever possible (38). There is no codified therapy. Apart from the prompt therapy of infections, recombinant human interferon, cautious use of anti-TNF in severe colitis, corticosteroids in obstructive inflammation, the bone marrow transplantation and genetic therapy are being taken in account (37).

Genetic testing may reveal combinations of several genes, thus explaining certain phenotypes or susceptibility to complications. For instance, co-existance of an early-onset sarcoidosis with a partial deficiency of interferon y receptor 1 (due to IFNyR1 defects) resulted in a mycobacterial infection induced by the BCG vaccination, as well as signs of macrophage activation syndrome after the initiation of tuberculostatic therapy (39).

\section{CONCLUSIONS}

The NOD2 associated autoinflammatory granulomatous diseases are an expanding group of disorders, with distinctive features. Identifying them may help better characterize the clinical phenotype in different populations and age groups, and also improve the therapeutic approach. Molecular data are crucial to better understand the disease and its management, to improve patients' outcome.

\section{Acknowledgments}

All authors have equal contribution. The authors would like to thank to Cristina Vulturar for technical support. 


\section{REFERENCES}

1. Yao Q, Li E, Shen B. Autoinflammatory disease with focus on NOD2-associated disease in the era of genomic medicine. Autoimmunity. 2019;52(2):48-56.

2. De Bruyn M, Vermeire S. NOD2 and bacterial recognition as therapeutic targets for Crohn's disease. Expert Opin Ther Targets. 2017;21(12):1123-39.

3. Yao Q. Nucleotide-binding oligomerization domain containing 2: structure, function, and diseases. Semin Arthritis Rheum. 2013;43(1):125-30.

4. Wouters CH, Maes A, Foley KP, Bertin J, Rose CD. Blau syndrome, the prototypic auto-inflammatory granulomatous disease. Pediatr Rheumatol Online J. 2014;12:33.

5. Mizuno N, Kume K, Nagatani Y, et al. Aggressive periodontitis and NOD2 variants. J Hum Genet. 2020;65(10):841-46.

6. Shen M, Moran R, Tomecki KJ, et al. Granulomatous disease associated with NOD2 sequence variants and familial camptodactyly: An intermediate form of NOD2-associated diseases?. Semin Arthritis Rheum. 2015;45(3):357-60.

7. Domínguez-Martínez DA, Núñez-Avellaneda D, Castañón-Sánchez CA, Salazar MI. NOD2: Activation During Bacterial and Viral Infections, Polymorphisms and Potential as Therapeutic Target. Rev Invest Clin. 2018;70(1):18-28.

8. Rosé $C D$, Pans $S$, Casteels I, et al. Blau syndrome: cross-sectional data from a multicentre study of clinical, radiological and functional outcomes. Rheumatology (Oxford). 2015;54(6):1008-1016.

9. Szymanski AM, Ombrello MJ. Using genes to triangulate the pathophysiology of granulomatous autoinflammatory disease: NOD2, PLCG2 and LACC1. Int Immunol. 2018;30(5):205-13.

10. Mensa-Vilaro A, Cham WT, Tang SP, et al. Brief Report: First Identification of Intrafamilial Recurrence of Blau Syndrome due to Gonosomal NOD2 Mosaicism. Arthritis Rheumatol. 2016; 68(4):1039-44.

11. International Society for Systemic Autoinflammatory Fevers. Infevers: database for hereditary autoinflammatory disorders mutations. Available at: http://fmf.igh.cnrs.fr/ISSAID/infevers.

12. Sarens IL, Casteels I, Anton J, et al. Blau syndrome-associated uveitis: preliminary results from an international prospective interventional case series. Am J Ophtalm. 2018;187:158-66.

13. Meiorin SM, Espada G, Costa CE, et al. Granulomatous nephritis associated with R334Q mutation in NOD2. J Rheumatol. 2007;34(9):1945-47.

14. Simonini G, Xu Z, Caputo R, et al. Clinical and transcriptional response to the long-acting interleukin-1 blocker canakinumab in Blau syndrome-related uveitis. Arthritis Rheum. 2013;65(2):513-8.

15. Chen J, Luo Y, Zhao M, Wu D, Yang Y, Zhang W, Shen M. Effective treatment of TNF $\alpha$ inhibitors in Chinese patients with Blau syndrome. Arthritis Res Ther. 2019;12;21(1):236.

16. Yao $Q$, Shen B. A systematic analysis of treatment and outcomes of NOD2-associated autoinflammatory diseas. Am J Med. 2017;130(3):365 e13-e18.

17. McDonald C, Shen M, Johnson EE, et al. Alterations in nucleotidebinding oligomerization domain 2- expression, pathway activation, and cytokine production in Yao syndrome. Autoimmunity. 2018; 51(2):53-61.

18. Dziedzic M, Marjańska A, Bąbol-Pokora K, et al. Co-existence of Blau syndrome and NAID? Diagnostic challenges associated with presence of multiple pathogenic variants in NOD2 gene: a case report. Pediatr Rheumatol Online J. 2017;15(1):57.

19. Gorevic $P$, Yao Q. Yao syndrome: a case report \& clinical review. The Rheumatologist, 2020. Available at: https://www.the-rheumatologist. org/article/yao-syndrome-a-case-report-clinical-review.
20. Yao Q, Piliang M, Nicolacakis K, et al. Granulomatous pneumonitis associated with adult-onset blau-like syndrome. American Journal of Respiratory and Critical Care Medicine. 2012;186(5):465-6.

21. Caso F, Gallozzi P, Costa L, et al. Autoinflammatory granulomatous diseases: from Blau syndrome and early-onset sarcoidosis to NOD-2 mediated disease and Crohn's disease. RMD Open. 2015;1(1):e000097.

22. Navetta-Modrov B, Ghebrehiwet B, Yao Q. Yao syndrome: A potential role and association of vasoactive intestinal peptide with NOD2. Rheumatology and Immunology Research. 2021;2(1):57-9.

23. Yao $Q$, Shen M, McDonald C, et al. NOD2-associated autoinflammatory disease: a large cohort study. Rheumatology (Oxford). 2015;54(10):1904-12.

24. Yao Q. Therapeutic Value of Canakinumab in Patients with Yao Syndrome. Arthritis Rheumatol. 2018;70 (suppl 10).

25. Horowitz JE, Warner N, Staples J, et al. Mutation spectrum of NOD2 reveals recessive inheritance as a main driver of early onset Crohn's disease. Sci Rep. 2021;11(1):5595.

26. Posovszky C, Pfalzer V, Lahr G, et al. Age-of-onset-dependent influence of NOD2 gene variants on disease behaviour and treatment in Crohn's disease. BMC Gastroenterol. 2013;13:77.

27. Bello GA, Adrianto I, Dumancas GG, et al. Role of NOD2 Pathway Genes in Sarcoidosis Cases with Clinical Characteristics of Blau Syndrome. Am J Respir Crit Care Med. 2015;192(9):1133-35.

28. Hamzaoui K, Abid H, Berraies A, et al. NOD2 is highly expressed in Behçet disease with pulmonary manifestations. J Inflamm (Lond). 2012;9(1):3.

29. Deng B, Ye Z, Li L, et al. Higher Expression of NOD1 and NOD2 is Associated with Vogt-Koyanagi-Harada (VKH) Syndrome But Not Behçet's Disease (BD). Curr Mol Med. 2016;16(4):424-435.

30. Kim HW, Kwon YJ, Park BW, et al. Diferential expression of NOD2-like receptors and their association $s$ with inflammatory responses in rheumatoid arthritis. Clin Exp Rheumatol. 2017;35(4):630-7.

31. Negroni A, Piedomenico M, Cucciara S, et al. NOD2 and inflammation: current insights. J Inflamm Res. 2018;11:49-60.

32. Macaluso F, Nothnagel M, Parwez Q, et al. Polymorphisms in NACHT-LRR (NLR) genes in atopic dermatitis. Experimental Dermatology. 2007;16:692-8.

33. Kutikhin AG. Role of NOD1/CARD4 and NOD2/CARD15 gene polymorphisms in cancer etiology. Human Immunology. 2011;72(10):955-68.

34. Beck DB, Aksentijevich I. Biochemistry of Autoinflammatory Diseases: Catalyzing Monogenic Disease. Front Immunol. 2019;10:101.

35. Zhou Q, Lee GS, Brady J, et al. A hypermorphic missense mutation in PLCG2, encoding phospholipase $\mathrm{C} \gamma 2$, causes a dominantly inherited autoinflammatory disease with immunodeficiency. Am J Hum Genet. 2012;91(4):713-20.

36. Aderibigbe OM, Priel DL, Lee CC, et al. Distinct Cutaneous Manifestations and Cold-Induced Leukocyte Activation Associated With PLCG2 Mutations. JAMA Dermatol. 2015;151(6):627-634.

37. Roos D. Chronic granulomatous disease. Br Med Bull. 2016; 118(1):50-63.

38. Papa R, Penco F, Volpi S, et al. Actin remodeling defects leading to autoinflammation and immune dystregulation. Front Immunol. 2021;11:604206

39. Cakan M, Kesindemirci G, Aydogmus C, et al. Coexistence of early onset sarcoidosis and partial interferon-y receptor 1 deficiency. Turkish J Ped. 2016;58:545-9.

Conflict of interest: none declared Financial support: none declared 